\section{LA COMISIÓN DE MUJERES Y CIENCIA DEL CSIC: DIEZ AÑOS PROMOVIENDO LA IGUALDAD DE OPORTUNIDADES Y LA EXCELENCIA EN EL ORGANISMO}

Pilar López Sancho, Joaquina Álvarez-Marrón, Flora de Pablo, Josefa Masegosa Gallego, Ma Carmen Mayoral Gastón, Elena Molina Hernández, Eulalia Pérez Sedeño, Francisca Puertas Maroto, Luisa Mạ Sandalio González

Cómo citar este artículo/ Citation: López Sancho, P.; ÁlvarezMarrón, J.; de Pablo, F.; Masegosa Gallego, J.; Mayoral Gastón, M. C.; Molina Hernández, E. et al. (2013). La Comisión de Mujeres y Ciencia del CSIC: diez años promoviendo la igualdad de oportunidades y la excelencia en el organismo. Arbor, 189(759):a012. doi: http://dx.doi.org/10.3989/arbor.2013.759n1011

Recibido: 5 diciembre 2012; Aceptado: 21 enero 2013.

RESUMEN: La Comisión Mujeres y Ciencia del Consejo Superior de Investigaciones Científicas (CSIC) fue creada en 2002 como comisión asesora de la Presidencia para temas de género. En sus diez años de funcionamiento, la comisión ha contribuido a mejorar la carrera científica de las mujeres investigadoras del CSIC y a aumentar la visibilidad de los resultados de sus investigaciones. En este trabajo se resumen los orígenes y objetivos de esta comisión, así como las actividades realizadas desde 2002, y se analiza la historia de la plantilla investigadora en ese periodo. Se recogen, también, los cambios producidos desde 2005 en la legislación española y europea en materia de igualdad de género. Al analizar específicamente los datos de la situación de las mujeres en 2003 y en 2012 desagregados por áreas de conocimiento, se muestra un avance en la proporción de mujeres en casi todas las áreas pero se está aún lejos del objetivo de paridad. El déficit en la presencia de mujeres es mayor en ciertas áreas y en el nivel profesional más alto, por lo que siguen siendo necesarias medidas activas para promover la igualdad, lo que a su vez contribuirá a la excelencia en las actividades científicas del CSIC.

PALABRAS CLAVE: ciencia y sociedad; igualdad de género.
THE WOMEN AND SCIENCE COMMITTEE OF THE SPANISH NATIONAL RESEARCH COUNCIL: TEN YEARS PROMOTING GENDER EQUALITY AND EXCELLENCE

Copyright: (C) 2013 CSIC. Este es un artículo de acceso abierto distribuido bajo los términos de la licencia Creative Commons Attribution-Non Commercial (by-nc) Spain 3.0.

ABSTRACT: The Women and Science Committee was set up in 2002 as an advisory unit on gender issues to the Presidency of the Spanish National Research Council (CSIC). During these ten years of existence, the committee has helped to improve the scientific careers of women in the CSIC and at the same time promoted the visibility of their scientific contributions. The history, objectives and activities of the Women and Science Committee during these years are reported here. The main gender policies and legal-framework changes at European and Spanish levels are recapped. Specifically, the sex distribution of permanent staff scientists in 2003 and 2012 are analysed and compared. The sex-disaggregated data by fields of knowledge show an improvement on the number and rank of female researchers in recent years, although they are still far from balanced. There is a bigger shortage of women in certain fields and at the top of the professional ladder. The implementation and monitoring of gender equality policies is still needed in order to approach a gender-balanced situation. This, in turn, will contribute to the excellence of the results of the CSIC's research activities. 


\section{Algo de Historia}

El 30 de septiembre de 2002 la Junta de Gobierno del Consejo Superior de Investigaciones Científicas, con informe favorable del Comité Científico Asesor y a propuesta de su Presidente, Rolf Tarrach, aprobó la creación de la Comisión Mujeres y Ciencia (CMYC). Sería una comisión para asesorar a la Presidencia en el estudio, seguimiento y optimización de la carrera científica de las mujeres en el CSIC. La CMYC está compuesta por ocho representantes electas de las correspondientes áreas en que se estructuran las actividades científicas del CSIC y otros cuatro vocales designados por la Presidencia. Buena parte del impulso generador de cierto progreso respecto a la igualdad de género en la carrera científica en el CSIC en los últimos años se debe a la creación de esta comisión. En este artículo queremos conmemorar los diez años de su existencia, recordar sus comienzos, constatar alguno de sus logros y resaltar los retos que todavía afrontan muchas de las áreas científicas para incorporar plenamente a las mujeres a la carrera investigadora. Entre los principales logros, está la publicación anual de los datos desagregados por sexos del personal científico que permiten hoy analizar cuál ha sido la evolución en todos estos años (ver http://www.csic.es/web/guest/ mujeres-y-ciencia). Además, la CMYC ha monitorizado la composición de los tribunales responsables de dirimir el acceso y la promoción del personal investigador, realizando un seguimiento sistemático de los resultados en la obtención de plazas, con el fin de determinar si han existido sesgos que causen menoscabo en el progreso de las mujeres en su carrera científica. Otro logro importante de la CMYC ha sido promover la visibilidad de las investigadoras y sus aportaciones científicas, incluyendo la divulgación de perfiles de prestigiosas investigadoras en su web.

La CMYC surgió a raíz de la celebración del Día Internacional de las Mujeres, en 2001, con un acto organizado por el Ministerio de Trabajo y Asuntos Sociales, a través del Instituto de la Mujer. Consistió en el Seminario "El papel de las mujeres españolas en la Ciencia" y la presentación de la Exposición "La Otra Mitad de la Ciencia" en los que las protagonistas fueron las mujeres científicas de nuestro país. Se celebró en la Sede del CSIC, en la calle Serrano 117 en Madrid. En la inauguración participaron el Ministro de Trabajo y Asuntos Sociales, Juan Carlos Aparicio y las Ministras de Educación, Cultura y Deportes, Pilar del Castillo; Sanidad y Consumo, Celia Villalobos y Ciencia y Tecnología, Anna M. Birulés (esta sólo en forma de vídeo pregrabado). También estuvieron presentes la Secretaria General de Asuntos Sociales, Concepción Dancausa, el Presidente del Consejo Superior de Investigaciones Científicas, Rolf Tarrach y la Directora General del Instituto de la Mujer, Pilar Dávila.
En el debate posterior a las intervenciones, las numerosas investigadoras presentes en el salón de actos del CSIC, de forma espontánea, argumentaron y pusieron de manifiesto la sutil discriminación de género existente históricamente en el organismo, y en el ambiente científico-universitario del país en general. En aquel momento, algunas investigadoras ya habían denunciado el desequilibrio de género en la ciencia en los países del Sur de Europa (De Pablo, 2000), hecho avalado por estudios de expertos y expertas presentados en publicaciones nacionales e internacionales. El debate continuó informalmente e intensamente entre los grupos formados tras el acto. El Presidente, en un principio incrédulo, se mostró más tarde convencido de que existía un desequilibrio anómalo entre hombres y mujeres en la plantilla investigadora del CSIC que merecía ser analizado. Por ello, nombró en mayo de 2001 un Grupo de Trabajo que fue el germen de la CMYC. Poco después, se elaboró y publicó el primer informe de la plantilla científica del CSIC con estadísticas desagregadas por sexo (Secretaría General de Recursos Humanos, 2001).

Como parte del esfuerzo para obtener información de la situación que existía entonces, en julio de 2002 se publicó un número monográfico de la revista $A R$ BOR titulado "CIENCIA Y TECNOLOGÍA EN EL CSIC: UNA VISIÓN DE GÉNERO” (Fernández Vargas y Santesmases, eds., 2002), en el que participaron científicas de las ocho áreas de la institución. La presentación del volumen estaba escrita por el Presidente Tarrach (2002), y vale la pena recordar aquí uno de los últimos párrafos de esta:

¿Qué papel juega el hecho de que la mujer se resiste más a perseguir el éxito "a cualquier precio", como, creo que correctamente, se apunta en una de las contribuciones? ¿Por qué la ambición y exigencia se ven como algo positivo en los hombres y no con tan buenos ojos cuando se trata de una mujer? ¿Cambiar las mujeres o cambiar la ciencia? se pregunta otra de las contribuciones. Y mucho más- Lea y medite, el problema lo merece.

...Pero no estamos bien, o no estamos donde debiéramos. Haremos lo necesario para mejorar y mejoraremos, no lo dudamos.

El número de $A R B O R$ se presentó en la tradicional fiesta de verano que se venía celebrando en la sede central del CSIC, y el Presidente posó con las colaboradoras del número para una emblemática foto en las escaleras (Figura 1). Algo que puede parecer anecdótico, pero que tiene la importancia de haber sido la primera vez en la historia del organismo, que un Presidente se ocupaba de un problema muy antiguo, la discriminación por género en una de las mayores instituciones científicas del país. Aunque la presidencia de la CMYC la ostenta el Presidente del CSIC y el Presidente 
Tarrach presidió personalmente las reuniones de la comisión, ésta ha tenido una presidencia delegada.

Los datos sobre la plantilla científica del CSIC revelados por esa primera recopilación desagregada por sexo eran muy claros: las mujeres representaban el $31 \%$ del personal investigador permanente, pero eran únicamente el $13 \%$ en la escala superior, la de Profesores de Investigación (PI). Los datos anteriores a 2001 con los que contábamos fueron recogidos por la Secretaría General del CSIC en 1981, cuando ostentaba el cargo la Profesora Concepción Llaguno, y en un estudio posterior (Alcalá, 1996). En ellos, el porcentaje de mujeres en la escala de PI era del $8 \%$, imantenido desde 1970 sin cambios! De la comparación podemos concluir que pasar del $8 \%$ al $13 \%$ de presencia de mujeres en la categoría de PI costó más de treinta años cuando la proporción total de mujeres investigadoras era de aproximadamente el $30 \%$. Esta conclusión indicaba que la progresión hacia la participación igualitaria de hombres y mujeres era un proceso inaceptablemente lento. Esta situación no era exclusiva del CSIC, en el año 2001 los datos de la Universidad Pública española no eran más esperanzadores, con un porcentaje de Catedráticas de Universidad del 12,4\% (Pérez Sedeño, coord., 2003).

\section{CONTEXTO INTERNACIONAL}

Al iniciar sus trabajos, y con el fin de hacer recomendaciones que ayudasen a cambiar la situación en el CSIC, la CMYC hizo un repaso exhaustivo de las políticas en materia de igualdad de género iniciadas por otras instituciones académicas del mundo. En 1999 el Massachusetts Institute of Technology (MIT), siendo Decano del área de Ciencias Robert J. Birgeneau, nombró una comisión que presidió Nancy Hopkins, para estudiar la situación de las mujeres en el claustro científico. En el informe, publicado en Internet y titulado Committee on the Status of Women Faculty, se aceptaba abiertamente que las mujeres estaban discriminadas en la School of Science. Se proponían medidas para corregir esta injusticia, y se programaba un seguimiento para analizar el éxito de las medidas. Poco después, las universidades con más prestigio de Estados Unidos seguían el ejemplo del MIT y publicaban informes con conclusiones similares.

Resultó ser cierta la frase de la promotora del informe del MIT, Profesora Hopkins: "Cada generación de mujeres... empezaba creyendo que la discriminación por razón de género se resolvió en la generación anterior... pero poco a poco se iban dando cuenta de que las condiciones no son igualitarias...". Las mujeres dedicadas a la investigación en España, con la excepción de algunas pocas de las áreas de Humanidades y Ciencias Sociales, no se habían dado cuenta de los obstáculos que existían en las instituciones, aunque hacía tiempo que diferentes organizaciones nacionales e internacionales habían detectado y denunciado la discriminación que sufrían las mujeres en diversos ámbitos de la sociedad. A pesar de que la igualdad entre hombres y mujeres se incluyó en la Carta de las Naciones Unidas (26 de junio de 1945) y en la Declaración Universal de los Derechos Humanos (10 de diciembre de 1948), así como en los más importantes convenios legales sobre derechos humanos, políticos y civiles, económicos, sociales y culturales estableci-

Figura 1: El Presidente Rolf Tarrach con las editoras y coautoras del número de la revista ARBOR dedicado a una visión de género en el CSIC. Fotografía tomada en la fiesta de verano del CSIC, en Madrid, en julio de 2002.

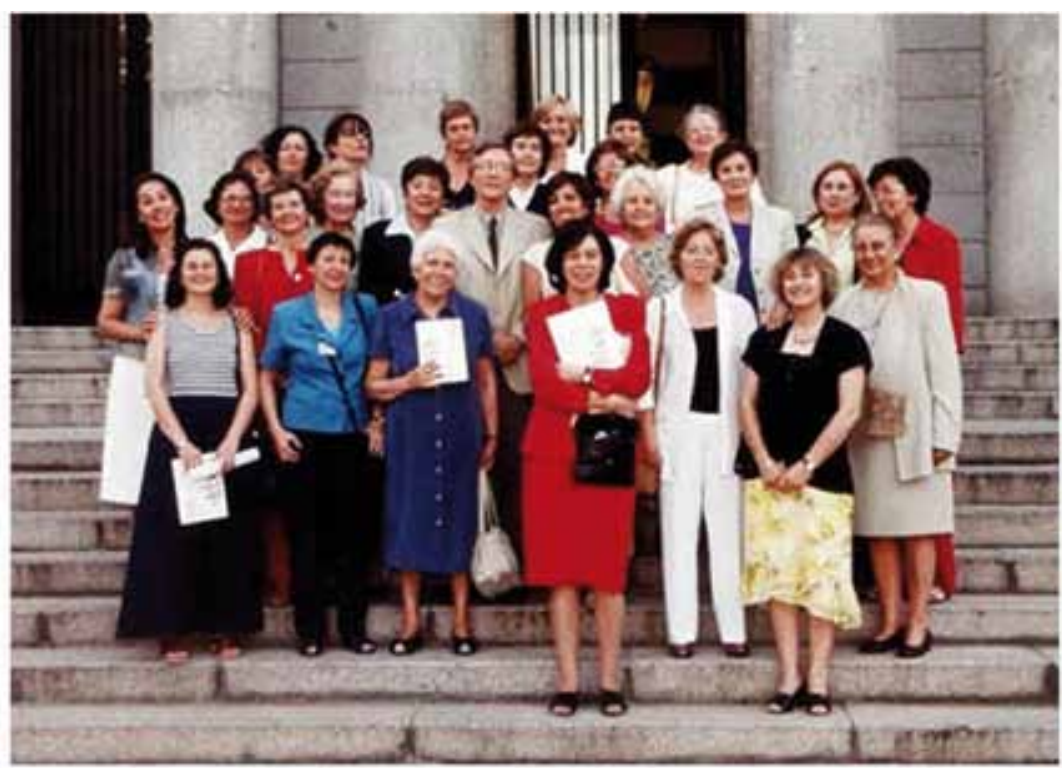


dos por las Naciones Unidas, hasta la década de los años setenta del pasado siglo XX, no se iniciaron las políticas contra las desigualdades de género a nivel internacional.

Los objetivos y medidas necesarios para conseguir la plena igualdad de género, tanto en la vida pública como en la privada, se definieron en 1979 en la Convención sobre la Eliminación de todas Formas de Discriminación contra las Mujeres (CEDAW) de la ONU. Durante la Década de las Mujeres de las Naciones Unidas (1975-85), se propusieron recomendaciones específicas para el ámbito científico, como por ejemplo, el programa de acciones titulado "Science and Technology, and Women" propuesto por el Panel del Comité Asesor sobre Ciencia y Tecnología para el Desarrollo de las Naciones Unidas en 1984. Desde entonces, en el continente americano, tanto en Estados Unidos como en Canadá, comenzaron a recopilar estadísticas separadas por sexo de forma sistemática en las instituciones académicas.

En Europa, en 1998, la Dirección General de Investigación creó un grupo de trabajo sobre las mujeres y la ciencia a nivel de toda la Unión Europea. Este grupo de expertas, elaboró el informe titulado: Política Científica de la Unión Europea. Promover la excelencia mediante la integración de la igualdad entre géneros, que se conoce popularmente como Informe ETAN (UE, 2000). El análisis de la situación de las mujeres en los sistemas de ciencia y tecnología de diversos países europeos, arrojaba la siguiente conclusión: "la infrarrepresentación de las mujeres amenaza los objetivos científicos de alcanzar la excelencia, además de ser un derroche y una injusticia". El Informe ETAN puso de manifiesto, además, la dificultad de obtener datos fiables sobre la participación de las mujeres en los sistemas de ciencia y tecnología. Por ese motivo, una de las recomendaciones del grupo ETAN fue que todos los estados miembros de la Unión Europea se comprometieran a elaborar estadísticas desglosadas por sexo y a hacerlas públicas. En el informe europeo de indicadores de ciencia y tecnología EC (2003), se incluyó una sección dedicada al análisis de la participación de las mujeres en la ciencia dentro de los países de la Unión. La Unión Europea mantiene una página web (http://europa.eu.int/comm/research/science-society/women/wssi/index_en.html) en la que se ofrecen informes, datos estadísticos y análisis. Prueba de la importancia que la Unión Europea da al tema género y ciencia, es la publicación periódica, desde 2003, de la serie She Figures con datos obtenidos en todos los países miembros (UE, 2003; 2006; 2009a, próximamente saldrá She Figures 2012) y en el esfuerzo que realiza al haber recopilado todos los documentos de la Comisión Europea al respecto en Stocking 10 years of women in science policy by the European Commission 1999-2009 (UE, 2009b).
Otras importantes sociedades e instituciones científicas se hicieron eco de las consecuencias significativas que tenía la escasez relativa de mujeres en muchas áreas científicas. Así, la Unión Internacional de Física Pura y Aplicada (conocida como IUPAP, por sus siglas en inglés) que engloba a todas las sociedades de física del mundo, organizó en marzo de 2002 en la sede de la UNESCO de París, la primera Conferencia Internacional de Mujeres en Física (ICWIP). La conferencia reunió representantes de 65 países y se analizaron las causas de la baja representación de mujeres que estudian y trabajan en los campos relacionados con las Ciencias Físicas. En ella se hicieron diferentes recomendaciones a los gobiernos e instituciones de docencia e investigación, sobre cómo eliminar los prejuicios y obstáculos que causaban el problema. En la actualidad, la mayoría de las sociedades científicas importantes cuentan con grupos o unidades que se ocupan de visibilizar la situación de las mujeres en los distintos ámbitos de la profesión correspondiente y tratar de mejorarla.

\section{Políticas de IGUALDAd EN ESPAÑA}

En marzo de 1910 se aprobó la "admisión de mujeres en todos los establecimientos docentes, sin necesidad de consultar a la Superioridad" que significó la apertura de la enseñanza superior y universitaria a las mujeres en España. En septiembre de ese mismo año se publicaba otra Real Orden por la que se establecía: "La posesión de los diversos Títulos Académicos habilitará a la mujer para el ejercicio de cuantas profesiones tengan relación con el Ministerio de Instrucción Pública". Esto posibilitó una incorporación paulatina de las españolas a la docencia e investigación (Magallón, 1998/2005).

En los años 30 del siglo XX, las mujeres eran casi el $9 \%$ del alumnado universitario español, llegando al $15 \%$ en los años 50 y a un $31 \%$ en los setenta (Flecha, 1996). Sin embargo, no hubo ninguna Catedrática de Universidad hasta 1953, cuando Ángeles Galino obtuvo la Cátedra de Historia de la Pedagogía de la Universidad de Madrid. Todavía en el siglo XXI, cuando las mujeres obtienen más del $60 \%$ de los títulos de grado universitarios, la proporción de mujeres en cátedras de universidad es inferior al 15,3\%, siendo el 37,3\% la proporción entre los profesores titulares de universidad. Se cumple el mismo patrón que en otros países, disminuyendo la proporción de mujeres cuando se avanza en la carrera académica. La situación estaba estancada a pesar del gran aumento de mujeres en el alumnado universitario en los últimos 30 años. Los documentos publicados por la Comisión Europea demostraban que el problema de la escasez de mujeres en las actividades científicas y su lento progreso en la carrera académica no se solucionaban solo con el paso del tiempo. Las recomendaciones dictadas por 
la Unidad de Mujeres y Ciencia, creada en 2001 por la Comisión Europea, indicaban la necesidad de atraer más mujeres a las ciencias experimentales y de eliminar los obstáculos que impedían su progreso. En este sentido, diversas normas jurídicas y directrices de la UE abogan por la conciliación real entre la vida profesional y familiar (todas las políticas y las aplicaciones de los fondos comunitarios han de ser coherentes con la defensa de la igualdad transversal). También instan a la adopción de acciones positivas (el principio de igualdad no impide el mantenimiento o la adopción de medidas que ofrezcan ventajas concretas a favor del sexo menos representado) y a la participación equilibrada en la toma de decisiones: la UE insta a los Estados a aprobar, si fuera necesario, medidas legislativas, reglamentarias y promocionales dirigidas a favorecer la participación equilibrada de hombres y mujeres en todos los ámbitos políticos, económicos y sociales. Finalmente resultan particularmente novedosos los criterios establecidos por el Tribunal de Justicia de la UE en torno a las discriminaciones indirectas, que conciernen de forma específica a las Universidades y Centros de investigación. El Tribunal de Justicia de la UE estableció con la llamada "inversión de carga de prueba" la necesidad de que sea la persona o institución demandada quien deba demostrar fehacientemente que no ha existido discriminación por razón de género.

La Constitución Española de 1978 establece en su Artículo 14 la igualdad de todos los españoles ante la ley: "Los españoles son iguales ante la ley sin que pueda prevalecer discriminación alguna por razón de nacimiento, raza, sexo, religión, opinión o cualquier otra condición o circunstancia personal o social". Garantizar su cumplimiento no es tarea fácil. La lucha de las mujeres fue importante en los años de la transición. Desde los años ochenta del siglo XX, existían asociaciones de mujeres que denunciaban la falta de igualdad y reclamaban medidas para erradicarla. Se fueron creando diversos Seminarios e Institutos de estudios de las mujeres o de género que dieron impulso a diferentes investigaciones, estudios, postgrados y doctorados, aunque el interés por la situación en el entorno de las ciencias exactas y naturales o por la tecnología era menor. En el ámbito científico universitario y de otros organismos había voces aisladas, que empezaron a ser más fuertes a finales de los años noventa, coincidiendo con las denuncias internacionales. En diciembre de 2001 se fundó la Asociación de Mujeres Investigadoras y Tecnólogas conocida por sus siglas, AMIT, con el objetivo principal de promover la igualdad de género en todos los ámbitos, particularmente en el de Ciencia y Tecnología. AMIT cuenta con cerca de 600 asociadas/os pertenecientes en su mayoría a universidades y organismos de investigación, y se extiende por toda la geografía española. Sus actividades se pueden consultar en (http://www.amit-es.org/) y ha colaborado activamente con la CMYC del CSIC en toda su andadura.

La primera medida legislativa específica en España para favorecer la igualdad entre mujeres y hombres, fue la publicación el 8 de marzo de 2005 de la Orden de Presidencia PRE/525/2005 firmada por María Teresa Fernández de la Vega, entonces Vicepresidenta del Gobierno y Ministra de la Presidencia. En esta Orden se impulsaban medidas que afectan, entre otros al empleo, a las empresas, al deporte, a la Administración General del Estado y a las Fuerzas Armadas. Estas medidas tienen también una aplicación específica a la investigación: se acordó la creación de una Unidad de Mujeres y Ciencia, entonces dependiente del Ministerio de Educación y Ciencia, "para abordar la situación de las mujeres en las instituciones científicas y mejorar su presencia en ellas"; también incluir, como criterio adicional de valoración en la concesión de ayudas a proyectos de investigación, la participación de mujeres en los equipos de trabajo. Otras medidas afectaban a los órganos de selección de personal de la Administración General del Estado y a los comités de expertos cuya composición tiene que contar con, al menos, un $40 \%$ de miembros del sexo menos representado.

Con posterioridad a 2005, diferentes leyes han incluido medidas en relación con la promoción de la igualdad entre mujeres y hombres en el ámbito de la investigación: la LOU, Ley Orgánica 4/2007, de 12 de Abril, de Universidades; la Ley de Igualdad, Ley Orgánica 3/2007, de 22 de Marzo, para la igualdad efectiva de mujeres y hombres; y por último la Ley de la Ciencia, la Tecnología y la Innovación de 2011 que significó un nuevo avance de las políticas proactivas para la consecución de la igualdad en el ámbito de la ciencia y la innovación. Esta ley, en su disposición adicional decimotercera, propone que: 1) los órganos, consejos y comités, así como los órganos de evaluación y selección del Sistema Español de Ciencia y Tecnología, se ajustarán a la composición y presencia equilibrada entre mujeres y hombres; 2) la incorporación de la perspectiva de género como una categoría transversal en la investigación y la tecnología, así como la promoción de los estudios de género y de las mujeres; 3) el Sistema recogerá, tratará y difundirá todos los datos desagregados por sexo e incluirá indicadores de presencia y productividad; 4) los procedimientos de selección y evaluación del personal investigador establecerán mecanismos para eliminar los sesgos de género que incluirán, siempre que sea posible, mecanismos de evaluación confidencial que impidan a la persona evaluadora conocer características personales de la persona evaluada, en particular su sexo y su raza; 5) la Estrategia Estatal de Innovación promoverá la incorporación de la perspectiva de género como una categoría transversal en todos los aspectos 
de su desarrollo; y 6) los Organismos Públicos de Investigación adoptarán Planes de Igualdad en un plazo máximo de dos años tras la publicación de esta ley, que serán objeto de seguimiento anual. Deberán incluir medidas incentivadoras para los que mejoren los indicadores por género en el correspondiente seguimiento anual.

La Unidad Mujeres y Ciencia (UMYC), inicialmente en el Ministerio de Educación y Ciencia y posteriormente en el de Ciencia e Innovación (ambos desaparecidos), publicó Académicas en cifras (2007), donde ofrecía los datos de todas las universidades públicas españolas, incluidos sus órganos de dirección. La UMYC, que está actualmente ubicada en la Secretaría de Estado de I+D+i del MINECO, (www.idi.mineco. gob.es/portal/site/MICINN/menuitem.7eeac5cd345 b4f34f09dfd1001432ea0/?vgnextoid=e218c5aa1649 3210VgnVCM1000001d04140aRCRD), ha publicado en 2011 Científicas en cifras y un estudio del sistema público de ciencia y tecnología desde una perspectiva de género, Libro Blanco, situación de las mujeres en la Ciencia Española. Este último se estructura según tres líneas de análisis, una dedicada a determinar las diferencias por género en la formación para la ciencia, la segunda enfocada a determinar las diferencias en la carrera científica y la tercera pretende determinar el papel de las instituciones en las diferencias por género. De todos estos estudios emana que la excelencia de las instituciones científicas mejoraría con un aprovechamiento óptimo de las capacidades de las mujeres dentro de su organigrama.

Desde la promulgación de la Ley de Igualdad, se han creado unidades y observatorios de igualdad en la mayoría de las universidades públicas españolas, se han elaborado planes de igualdad y se han implementado estudios de género. La Ley de Igualdad también establece que todos los ministerios cuenten con una unidad de igualdad que vigile el cumplimiento de sus artículos. La legislación española en materia de igualdad se situó entre las más avanzadas de Europa hasta 2010. Desafortunadamente, la falta de atención desde el inicio de la crisis económica ha provocado que España descienda 14 puestos en el índice global de igualdad de género durante el pasado año (Pereira, 2012).

\section{PREMIOS Y HONORES}

Uno de los temas más complejos en materia de igualdad es el concepto de "excelencia", que subyace en la concesión de premios o galardones al trabajo científico de superior calidad (Duarte, 2009). Todos los recientes premios a la "excelencia" en España han sido concedidos a hombres. En 2011, dentro del Programa de Fomento de Investigación, Desarrollo e Innovación que en la actualidad se enmarca en la Se- cretaría de Estado de Investigación, Desarrollo e Innovación del Ministerio de Economía y Competitividad, se convocaron cinco Premios Nacionales de Investigación correspondientes a: ciencias físicas, ciencias y tecnologías químicas, ciencias y tecnologías de los recursos naturales, matemáticas y tecnologías de la información y comunicaciones, y transferencia de las tecnologías (http://www.idi.mineco.gob.es/portal/ site/MICINN/menuitem.dbc68b34d11ccbd5d52ffeb8 01432ea0/?vgnextoid =5f2b6e1e125ae210VgnVCM10 00001d04140aRCRD).

En 2012, la Fundación Premios Rey Jaime I ha concedido siete galardones en las áreas: investigación básica; economía; investigación médica; protección del medio ambiente; nuevas tecnologías; urbanismo, paisaje y sostenibilidad; emprendedor; (http://www. fvea.es/premios_home.html\#).

Los Premios de la Fundación BBVA Fronteras del Conocimiento, con carácter internacional, se convocaron en 2011 en las categorías de ciencias básicas (física, química, matemáticas); biomedicina; ecología y biología de la conservación; economía, finanzas y gestión de empresas; música contemporánea; cambio climático; cooperación y desarrollo; (http://www.fbbva.es/ TLFU/tIfu/esp/microsites/premios/fronteras/index. jsp.)

En total, entre estos citados, han sido galardonados veintiún científicos con destacadas contribuciones al conocimiento. Todos ellos tienen en común la excelencia de su trabajo y además, como hemos dicho, todos son varones (López, 2012a). La baja proporción de científicas premiadas es un problema global, de hecho las mujeres han recibido un $5,3 \%$ del total de los Premios Nobel. Este desequilibrio hizo que en 1998 se crearan los Premios Internacionales L'OREAL-UNESCO Por las Mujeres en la Ciencia (http://www.unesco.org/ new/en/natural-sciences/priority-areas/gender-andscience/for-women-in-science-programme/2012awards/) dirigidos exclusivamente a mujeres, con el objetivo de "apoyar el progreso de las mujeres en la ciencia".

Las convocatorias de premios dirigidos únicamente a mujeres es un tema muy polémico en los ambientes científicos, ya que podrían indicar que existen dudas sobre la neutralidad en el sistema de valoración de méritos en otras convocatorias. Los datos citados justificarían una revisión de los métodos vigentes de evaluación, y de presentación de candidaturas. En línea con la innovación, de hecho, la Comisión Europea en colaboración con la Universidad de Standford tiene en marcha un proyecto titulado "Gendered Innovations" que incluye el análisis de género como fuente para la creación de nuevos conocimientos y tecnología (http://genderedinnovations.stanford.edu/what-isgendered-innovations.html). 
Algunos resultados de este proyecto ponen en evidencia los fallos del sistema vigente de valoración de méritos, fallos apuntados ya hace años (Wenneras y Wold, 1997) y que en años recientes solo se ha hecho más sutil pero no ha desaparecido. El problema tampoco es exclusivo del viejo continente. En un estudio realizado entre profesores de ambos sexos de universidades importantes de EEUU, publicado en la prestigiosa revista científica PNAS (Moss-Racusin et al., 2012), se concluye la existencia de "un sutil sesgo a favor de los estudiantes varones". La evaluación del mismo expediente académico por 127 profesores de Biología, Química y Física, resultó con valoraciones más altas cuando creían que pertenecía a un varón que cuando creían que pertenecía a una mujer. Es especialmente significativo el impacto del género del estudiante en las posibilidades de admisión y en el salario propuesto por los evaluadores. En conclusión, las mujeres, a igualdad de méritos, tenían menos probabilidades de ser contratadas porque eran consideradas menos competentes.

\section{ACtividades y APORTACIONES de la COMISIÓn MUJe- RES Y CIENCIA DEL CSIC}

En sus algo más de diez años de funcionamiento la CMYC ha cumplido sus objetivos principales: visibilizar los datos de personal desagregados por sexo, estudiar las posibles causas que hacen difícil tanto el ingreso como el progreso de las mujeres en la carrera científica y proponer a la Presidencia posibles acciones destinadas a conseguir la mayor igualdad entre mujeres y hombres en la carrera científica en el CSIC.

La elaboración del informe anual de la situación y el seguimiento y análisis de los resultados han estimulado el progreso de las mujeres en la carrera científica. La CMYC ha velado por el cumplimiento de las recomendaciones y en los tribunales de oposición y demás órganos de decisión del CSIC generalmente ya se cumple con la presencia equilibrada de ambos sexos (algo menos en las comisiones de área). Conviene, sin embargo, recalcar que la otra circunstancia que en la década contemplada probablemente ha contribuido a aumentar el número de mujeres totales, y su promoción a escalones más altos, ha sido el incremento en la oferta de plazas, particularmente entre 2005 y 2008.

La CMYC elaboró en 2005 una Acción Horizontal para la Equidad de Género y un Plan de Igualdad para la carrera investigadora que estuvo vigente entre 2007 y 2010. Más recientemente ha elaborado recomendaciones para el uso de lenguaje no sexista, todo ello siguiendo las recomendaciones de la Comisión Europea, y del extinto Ministerio de Igualdad. (documentos disponibles en la página web de la CMYC, www.csic.es/web/guest/informes-cmyc). Representantes de la CMYC asesoraron en temas de igualdad en la redacción del Estatuto de la Agencia Estatal CSIC en 2007. En colaboración con la Presidencia del CSIC, la CMYC ha trabajado para que en las Memorias anuales de los centros del CSIC se incluyan estadísticas desagregadas del personal, que permitan analizar los cambios en la situación de las mujeres en años sucesivos. Miembros de la CMYC han representado al CSIC en diferentes foros. En 2009, con motivo del décimo aniversario de los trabajos de la Comisión Europea en materia de igualdad, se celebró en Praga la Conferencia 'Changing research landscapes to make the most of human potential 10 years of EU activities in Women and Science and BEYOND', la CMYC presentó la ponencia 'Narrowing down gender gap in Spanish Scientific Institutions'.

Por invitación, la CMYC participó en el "Encuentro Nacional de Unidades de Igualdad de las Universidades Españolas", celebrado en la Universidad Internacional Menéndez Pelayo, en agosto de 2010. La CMYC estuvo representada en el European Gender Summit que tuvo lugar en Bruselas, en noviembre de 2011, reuniendo a más de 400 participantes. La Agencia Estatal CSIC era una de las instituciones que apoyaban esta iniciativa como Patrono. Es de destacar que entre las conclusiones de este importante encuentro está la necesidad de seguir luchando por la igualdad en los organismos científicos europeos.

Varias vocales de la comisión participaron como invitadas en el VIII Seminario de Investigación Interdisciplinar: Tecnologías, Ingeniería y Ciencias Experimentales: Rompiendo fronteras en los estudios de género, en el Instituto Universitario de Estudios de la Mujer (Universidad Autónoma de Madrid) en mayo de 2011. Con motivo de la celebración del centenario de la concesión a Madame Curie del Premio Nobel de Química (1911) la CMYC ha participado en diversos homenajes que han tenido lugar a lo largo de 2011.

A su vez la CMYC ha organizado diferentes actos como el dedicado a celebrar el centenario del libre acceso de las mujeres a la universidad española, "Mujeres y Ciencia, 100 años en la Universidad", organizado en colaboración con el Departamento de Divulgación Científica del CSIC y Presidencia de Gobierno, dentro del Programa Ellas Crean 2010. El acto tuvo lugar el 9 de marzo de 2010 en Madrid, en el salón de actos del CSIC.

La CMYC ha organizado actos encaminados a promover la visibilidad del trabajo de las mujeres como el Acto Académico celebrado en marzo de 2011, en homenaje a la Profesora Concepción Llaguno Marchena, del Área de Ciencia y Tecnología de Alimentos, fallecida en noviembre de 2010. En diciembre de 2011 se organizó, en colaboración con la representación del CSIC en Bruselas, el seminario "La perspectiva de género en la investigación". Viviane Willis-Mazzichi, Head of Gender Sector Unit "Ethics and Gender" B Di- 
rectorate European Research Area, impartió la conferencia "The Gender strategy in the European Research Policy", donde explicó los planes futuros de la Comisión Europea en materia de igualdad.

Fruto del trabajo de la CMYC, junto al mencionado incremento de plazas para el CSIC que tuvo lugar en la Oferta Pública de Empleo durante el periodo 2005-2008, la situación de las mujeres de la plantilla científica del CSIC ha experimentado una mejora considerable. Se ha alcanzado un $23,4 \%$ de mujeres en la categoría de Profesores de Investigación, muy cerca del mínimo óptimo fijado en los acuerdos de Lisboa para 2010 que era del 25\%, y ligeramente por encima de la media europea reflejada en las She Figures para la categoría más alta en las instituciones académicas.

En la gráfica de la Figura 2 se puede observar el progreso de la proporción de mujeres en la carrera investigadora del CSIC desde los estadios de formación hasta el nivel más alto, entre 2005 y 2010 . Este quinquenio representa el mejor de la historia de la institución para los avances en igualdad de género. Los datos corresponden a: personal en formación (becascontratos predoctorales), doctores contratados por el Programa Ramón y Cajal y las tres categorías de personal científico funcionario.

\section{SITUACIÓN DE LAS CIENTÍFICAS EN LAS DISTINTAS ÁREAS DE CONOCIMIENTO}

A continuación se representa la evolución en la proporción de mujeres y hombres investigadores de plantilla que ha tenido lugar, en cada área científica del CSIC, en los años de trabajo continuado de la CMYC. Se basa en la comparación de los datos recogidos en los informes anuales de los años 2003 (Recursos Humanos del CSIC, 2003) y 2012 (Mujeres y Ciencia, 2012). En el primer informe se recoge el personal de las tres escalas de investigadores permanentes que había en la primavera de 2003 y en el de 2012 los que había en Diciembre de 2011. En cada caso se dan las cifras de mujeres y hombres en las distintas escalas científicas, representados en valor absoluto en los histogramas. En estos histogramas, la abreviatura CT corresponde a la escala de Científicos Titulares; IC corresponde a la escala de Investigadores Científicos; y PI a la escala de Profesores de Investigación. También se presentan gráficamente para cada área los porcentajes relativos en las escalas que reflejan una imagen, habitualmente llamada "tijera", a medida que se asciende desde CT a PI. La variación porcentual entre los años 2003 y 2012 en todas las escalas esta así mismo ilustrada. Las gráficas van ordenadas siguiendo la numeración oficial de las áreas en el CSIC (ver Figuras 3 a 10).

Figura 2: Porcentaje de mujeres y hombres en los distintos niveles a lo largo de la carrera científica en el CSIC en 2005 y 2010. RyC, contratos Ramón y Cajal; CT, Científicos/as Titulares; IC, Investigadores/as Científicos; PI, Profesores/as de Investigación.

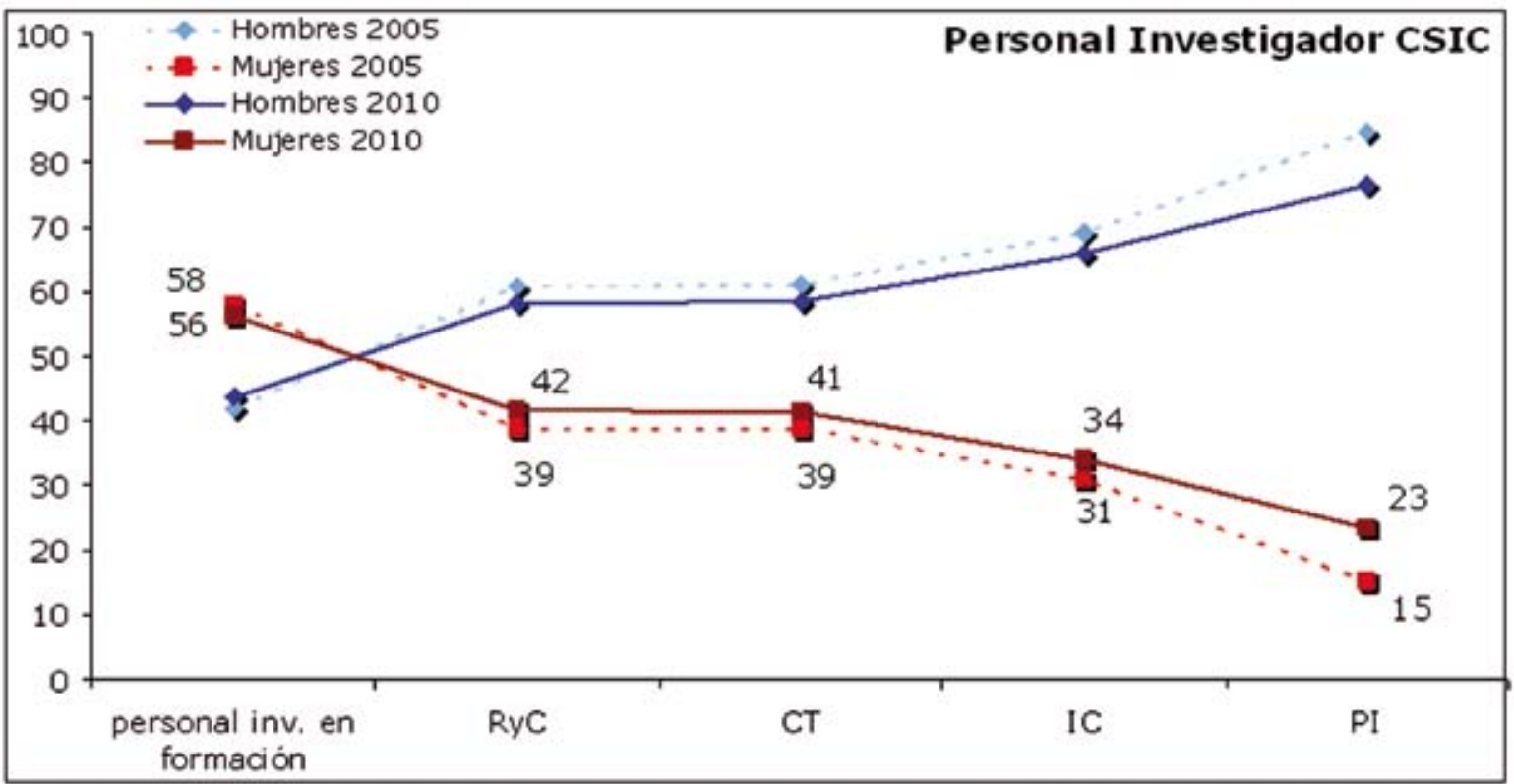


Figura 3. Personal científico del Área de Humanidades y Ciencias Sociales (I).
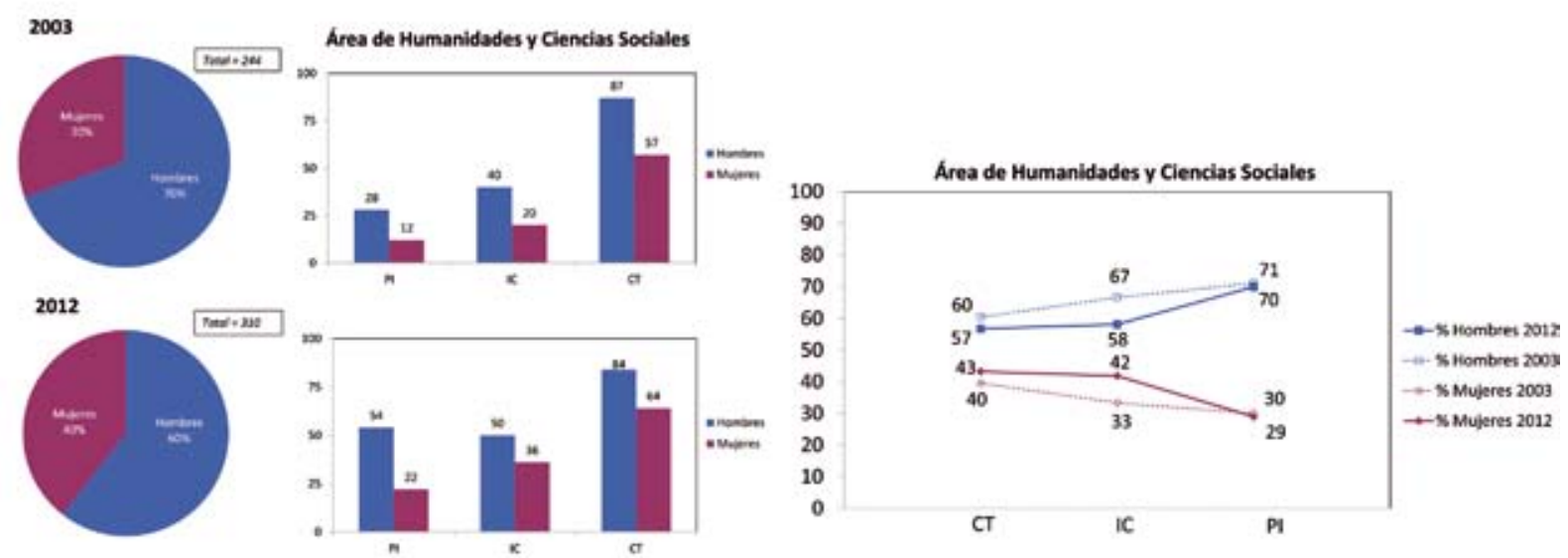

Figura 4. Personal científico del Área de Biología y Biomedicina (II).
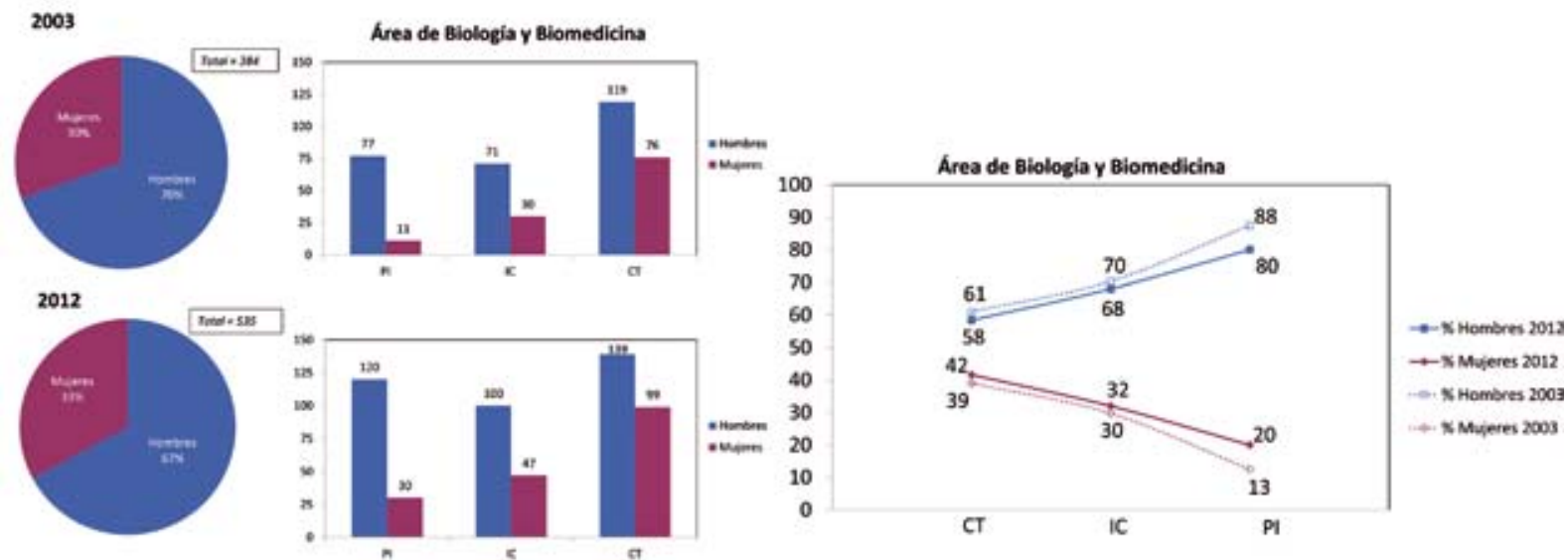

Figura 5. Personal científico del Área de Recursos Naturales (III).
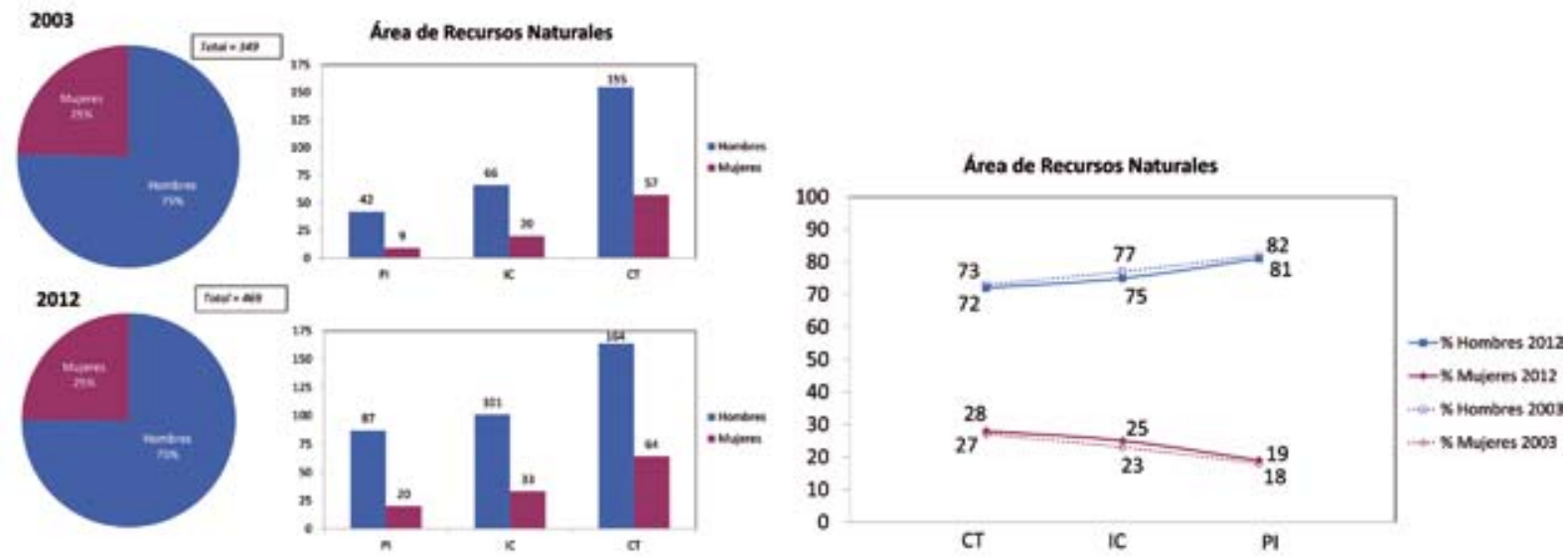
Figura 6. Personal científico del Área de Ciencias Agrarias (IV).
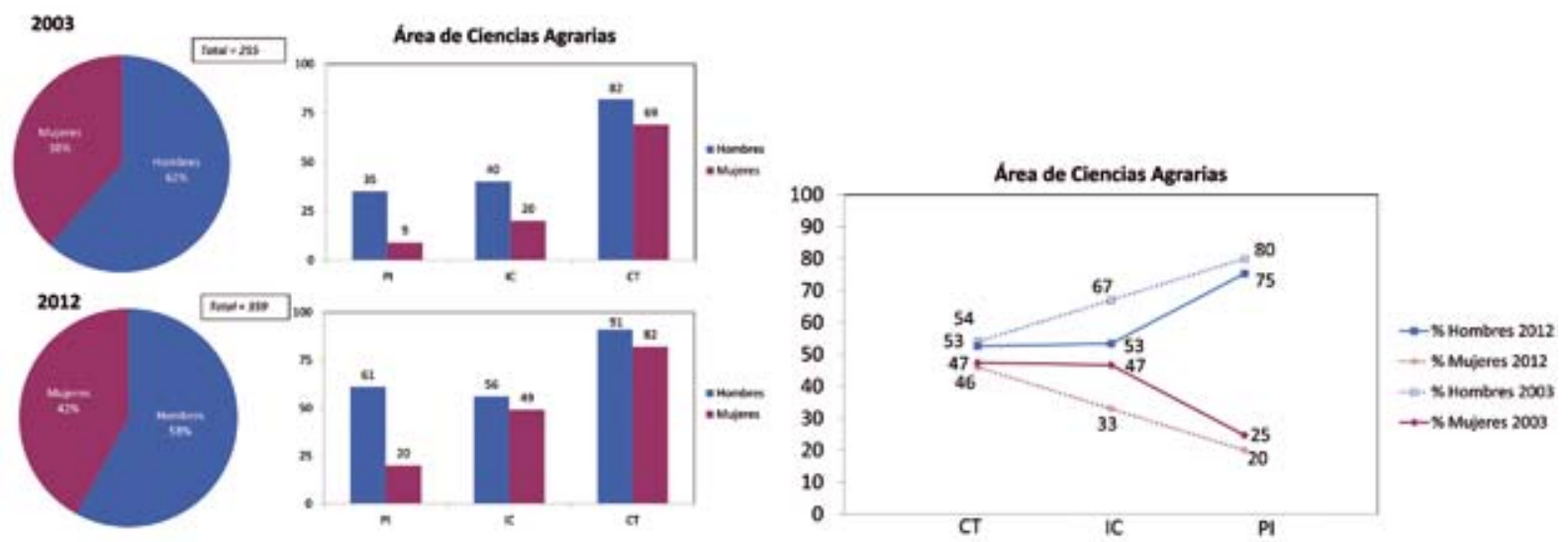

Figura 7. Personal científico del Área de Ciencia y Tecnologías Físicas (V).
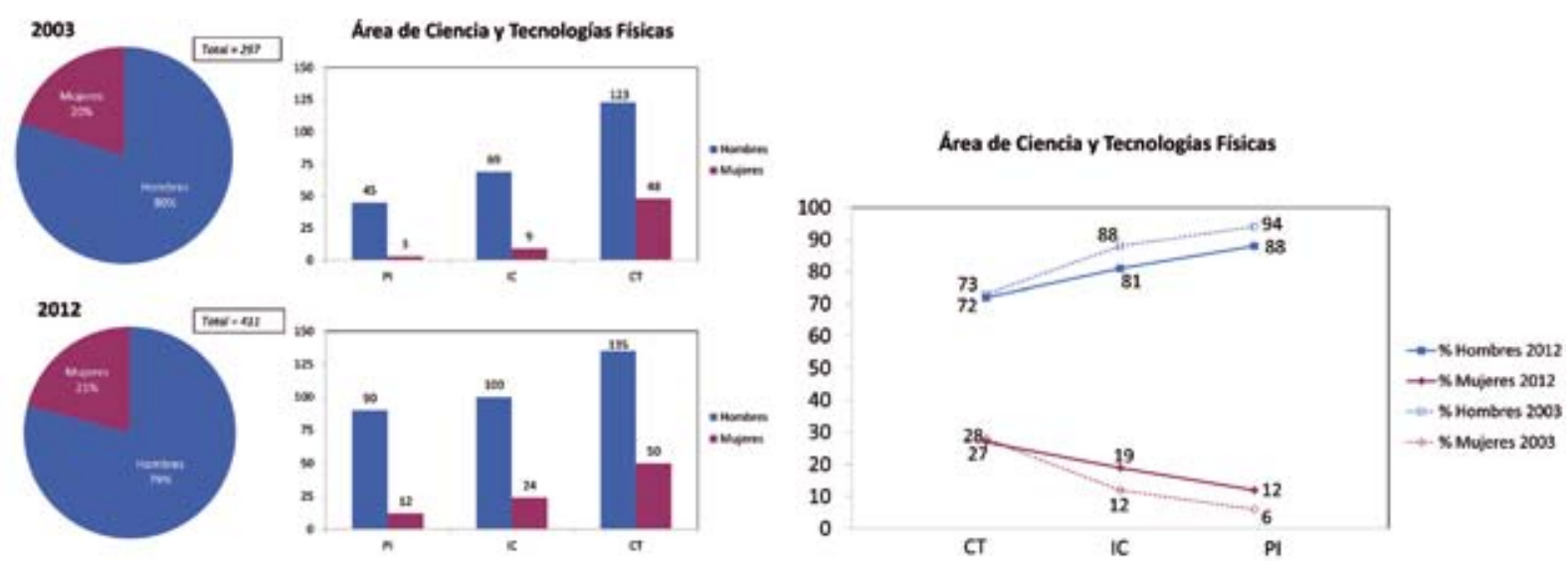

Figura 8. Personal científico del Área de Ciencia y Tecnología de Materiales (VI).
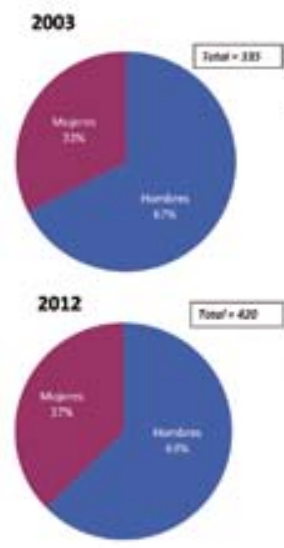

Area de Ciencia y Tecnologia de Materiales
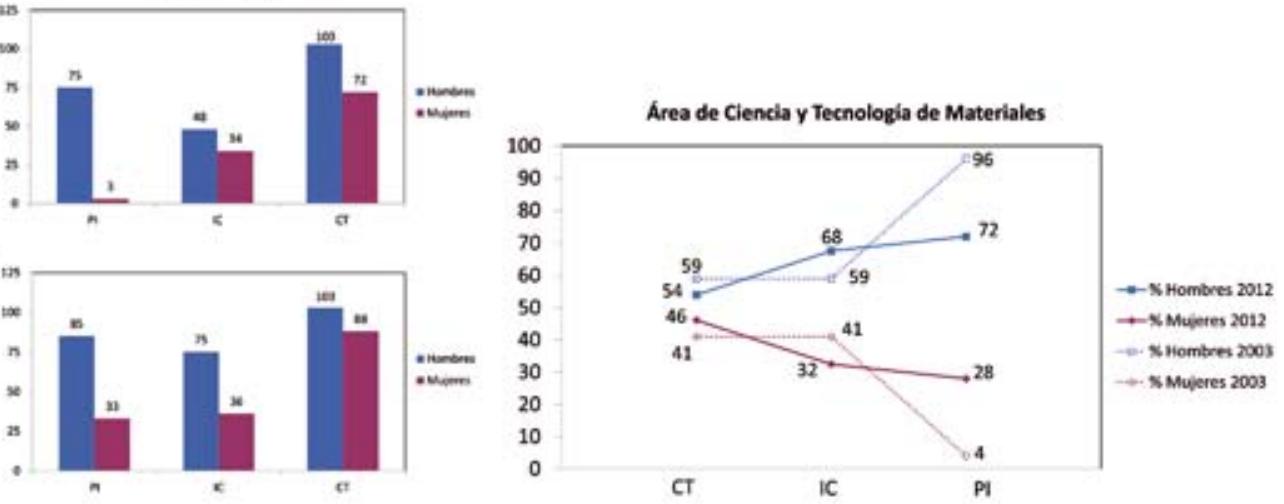
Figura 9. Personal científico del Área de Ciencia y Tecnología de Alimentos (VII).
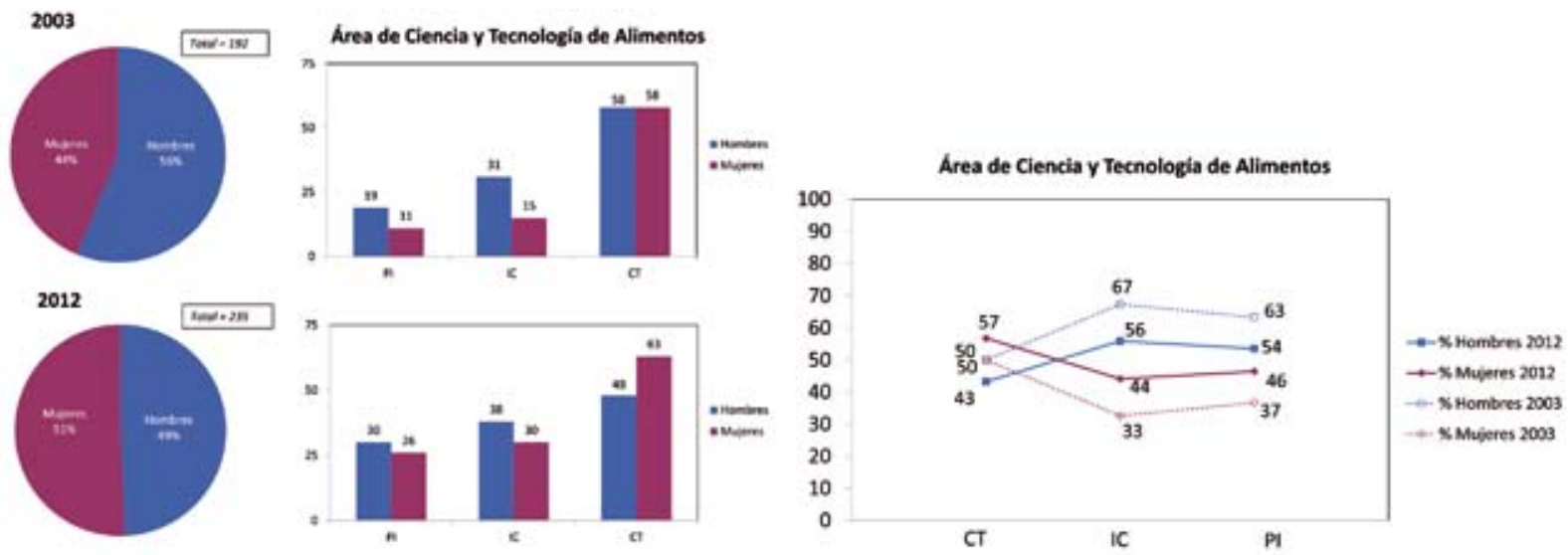

Figura 10. Personal científico del Área de Ciencia y Tecnologías Químicas (VIII).
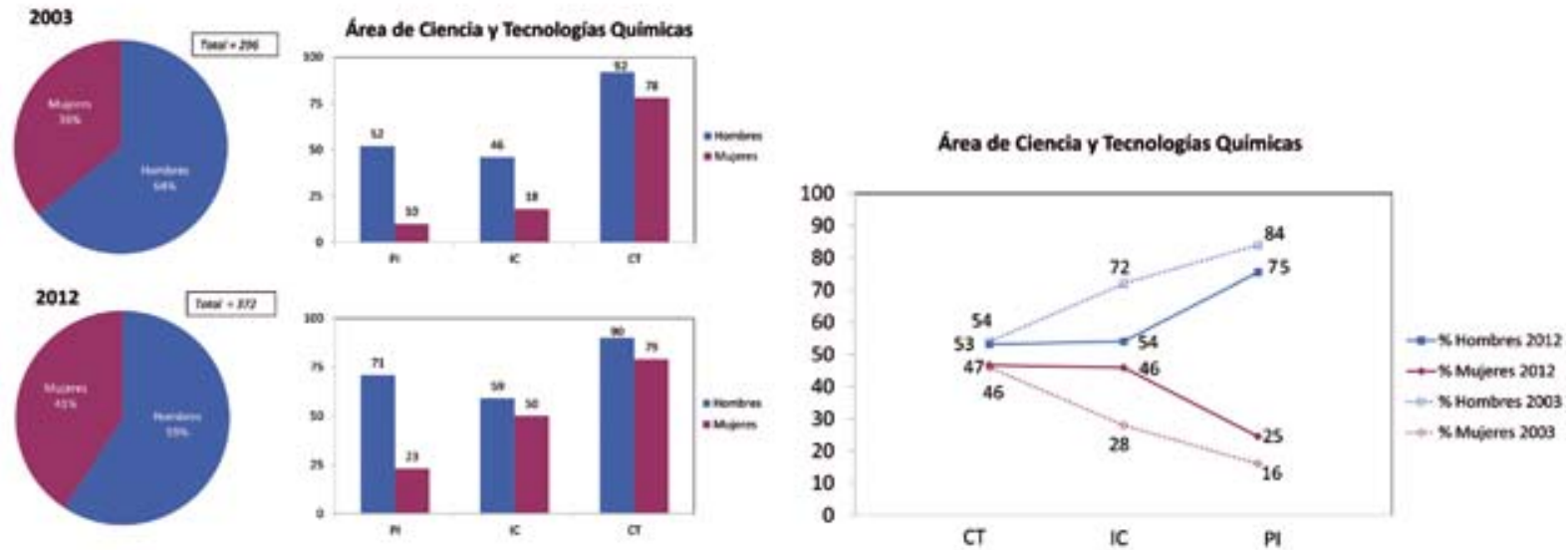

En conjunto, se observa una mayor incorporación de mujeres en todas las categorías científicas del CSIC, pero en algunas el avance es muy lento. El progreso más significativo ha sido en el área de C. y T. de Alimentos, la única que ha llegado a la paridad en las tres escalas. Este avance pudo estar facilitado por ser ésta el área más pequeña en personal investigador, con un total de 235 personas. También puede haber influido la existencia de un número importante de mujeres que han ido ocupando lugares de responsabilidad y visibilidad (Concha Llaguno, Manuela Juárez, etc.), lo que ha podido constituir un elemento tractor de la incorporación y promoción de mujeres en el área. También es significativo el progreso observado en C. Agrarias, que alcanza casi la paridad en las escalas de CT e IC y experimenta un incremento importante en la escala superior de $\mathrm{PI}$, situándose próximo al $25 \%$ recomendado en los acuerdos de Lisboa. La misma tendencia se observa en el área de C. y T. Químicas donde las mujeres representan el $46 \%$ de IC y el $25 \%$ de PI.

Hay que resaltar también el progreso claro en las áreas más técnicas, C. y T. de Materiales y C. y T. Físicas. El caso del cambio de pendiente entre las escalas de IC y PI en el área de Materiales es tan fuerte que seguramente significa que se "rompieron las barreras" existentes a la natural promoción, quizá como resultado de las acciones positivas realizadas a raíz de la existencia de la comisión. Bajo nuestro punto de vista, resultó esencial la paridad en los tribunales a partir de 2005 que significó, como muestran los informes de la época, el inicio de una casi equiparación de la ratio de éxito de mujeres y hombres en los accesos a las plazas. En el área de C. y T. Físicas, por otro lado, el número de científicas que han promocionado a IC y PI es relativamente significativo. Dado que se partía de una proporción tan baja de mujeres, los porcentajes alcanzados en las escalas superiores aún están bastante por debajo de la media de mujeres en el área, que desafortunadamente es la menor de todas, con un $21 \%$. Sin embargo sólo hay un aumento de un punto porcentual en esta década para CT. 
En el otro lado, datos desalentadores, hay una verdadera anomalía en la "pirámide natural" de las escalas de varones, tanto en la media de todas las áreas, como en Humanidades, Biomedicina, Agrarias, Materiales, y Químicas. Es decir, en todos estos casos hay mayor número absoluto de hombres PI que de IC. Eso indica una "subida acelerada" a la escala superior que no ocurre en las escalas de las mujeres.

Las áreas donde el progreso ha sido muy inferior al esperable son Humanidades y Ciencias Sociales, Recursos Naturales y Biomedicina. En la primera, sorprende el prácticamente nulo aumento en las profesoras de investigación, aunque las investigadoras han subido algo más de ocho puntos en el mismo periodo, en un área muy feminizada. En Recursos Naturales el inmovilismo en las tres escalas es dramático y habrá que plantear un análisis en profundidad y medidas proactivas correctoras específicas para equilibrar la presencia de hombres y mujeres. Esta área es la segunda por tamaño, con 469 investigadores de plantilla de los que solamente el $25 \%$ son mujeres. Esta proporción se ha mantenido, incluso cuando ha habido un aumento neto en 120 investigadores. Esto es especialmente sorprendente dado que temáticamente se nutre de licenciados/as en titulaciones universitarias con una alta presencia de mujeres, como es el caso de la Biología. En la evolución de esta área, también es destacable el importante incremento de los varones de la escala de Profesores de Investigación, de manera que actualmente representan el $25 \%$ del total de investigadores permanentes varones en el área. El caso del área de Biomedicina es también preocupan- te, el progreso de promoción a IC ha sido lento (poco más de $2 \%$ relativo) y aunque hay un mejoría clara del número de mujeres dentro de la escala de PI (el porcentaje ha pasado de $12,5 \%$ a $20 \%$ ), hay una notable distancia todavía respecto a la media de mujeres en el área, ahora del $33 \%$, pero ya desde hace varias décadas por encima del $30 \%$. Que se haya dado la "subida acelerada" de varones a la escala superior, y que sea el área más grande, con 535 personas en la plantilla científica, pudieron jugar un papel en esta inercia que dificulta conseguir la paridad en escalones superiores, caso opuesto al del área de Alimentos.

\section{RETOS FUTUROS}

A pesar de los avances reseñados tanto en el marco jurídico español como en las normativas de los diferentes organismos, entre los que destacamos el CSIC, existen muchas sombras aún en el funcionamiento de las instituciones en materia de igualdad. El principio de representación equilibrada por sexos en las comisiones generales designadas por la Presidencia no se cumple y, a pesar de estar en la ley, crea polémica. Esta asimetría está presente en las Comisiones de Área y en la Comisión de Ética, algo parecido a lo que ha ocurrido con la reciente Directiva Europea, conocida como Ley Reding, que impone que las empresas europeas cotizadas cuenten en 2020 con un $40 \%$ de consejeras. La presencia de mujeres en los espacios de poder sigue provocando reacciones airadas. En el CSIC también está lejos de la paridad el número de mujeres directoras de centros e institutos.

Figura 11. Datos desagregados por sexo de los directores y los consejos de redacción de las revistas editadas por el CSIC.

\section{REVISTAS CIENTÍFICAS DEL CSIC}

\section{7 revistas científicas del CSIC \\ 11 Ciencia y Tecnología \\ 4 Ciencias Sociales 22 Arte y Humanidades}
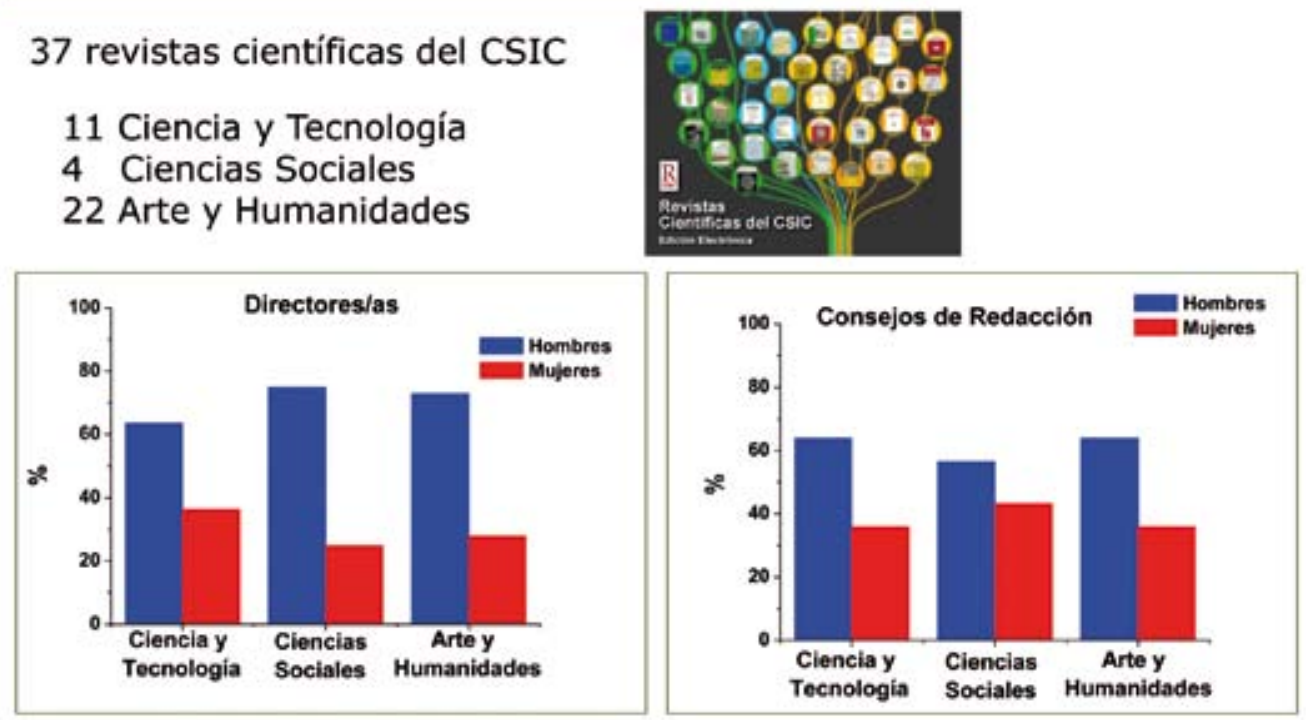
La falta relativa de mujeres no solo se da en los ámbitos mencionados en el CSIC. En los comités científicos de los congresos, entre los conferenciantes plenarios o en los consejos de redacción de las revistas científicas de todo el mundo académico español, las mujeres son muy minoritarias. En la Figura 11 se presenta como muestra los datos del sexo de los directores y la composición de los consejos de redacción de las 37 revistas propias del CSIC. Por todo ello, tenemos aún muchos retos por delante, de los que queremos resaltar los siguientes:

a) Aumentar el número de científicas que entran al CSIC, particularmente en áreas como C. y T. Físicas o Recursos Naturales, exigirá divulgación y abolición de estereotipos sexistas actuando en centros educativos tempranos.

b) Seguir monitorizando el progreso de las CT a IC $y$, sobre todo, de estas últimas a $\mathrm{PI}, \mathrm{y}$ reiterar la necesidad de que el apoyo institucional (de los centros e institutos, no solo del organismo) sea equivalente al recibido por colegas masculinos para la obtención de espacios, personal técnico de apoyo, etc.

c) Vencer la resistencia de los y las colegas que creen que el problema de desigualdad de oportunidades y visibilidad no existe para las mujeres en el CSIC, algunos de los cuales ocupan puestos de responsabilidad. Exigir que se apliquen las recomendaciones de la Presidencia en materia de género en todos los centros como, por ejemplo, que las Memorias científicas incluyan los datos de personal desagregados por sexo.
En definitiva, tenemos que hacer del CSIC una institución donde las mujeres deseen trabajar y ambicionen progresar, sin encontrarse más (tampoco menos) dificultades que los hombres de su generación. En la Figura 12 se presenta la evolución de la plantilla investigadora en los últimos diez años en números absolutos, el aumento de mujeres en la institución es lento y el número sigue siendo muy bajo, a pesar del considerable incremento de mujeres con becas y contratos. Nuestro análisis indica que estamos progresando, aunque aún no hemos llegado a la meta.

\section{CONCLUSIONES}

El Consejo Superior de Investigaciones Científicas ha realizado durante los pasados años un gran esfuerzo por modernizarse y mejorar su calidad. La lucha por alcanzar la igualdad está enmarcada en este afán de progreso y consolidación de la excelencia. Desafortunadamente, la desigualdad entre la participación de mujeres y hombres sigue siendo un hecho y una debilidad en nuestra institución. Un problema que afecta a la sociedad y que está presente en la mayoría de las instituciones científicas a nivel global, puesto de manifiesto por los estudios realizados por expertas y expertos. A pesar de la legislación específica y de las políticas de igualdad internacionales, el progreso de las mujeres en las instituciones científicas es mucho más lento de lo que cabría esperar. Esto ha hecho que las políticas europeas hayan variado su enfoque: hay que cambiar el funcionamiento de los organismos y detectar los fallos estructurales que frenan a las investigadoras (López, 2012b). Por ello, la Comisión Europea dirige sus

Figura 12.- Variaciones del personal investigador funcionario del CSIC durante los últimos diez años.

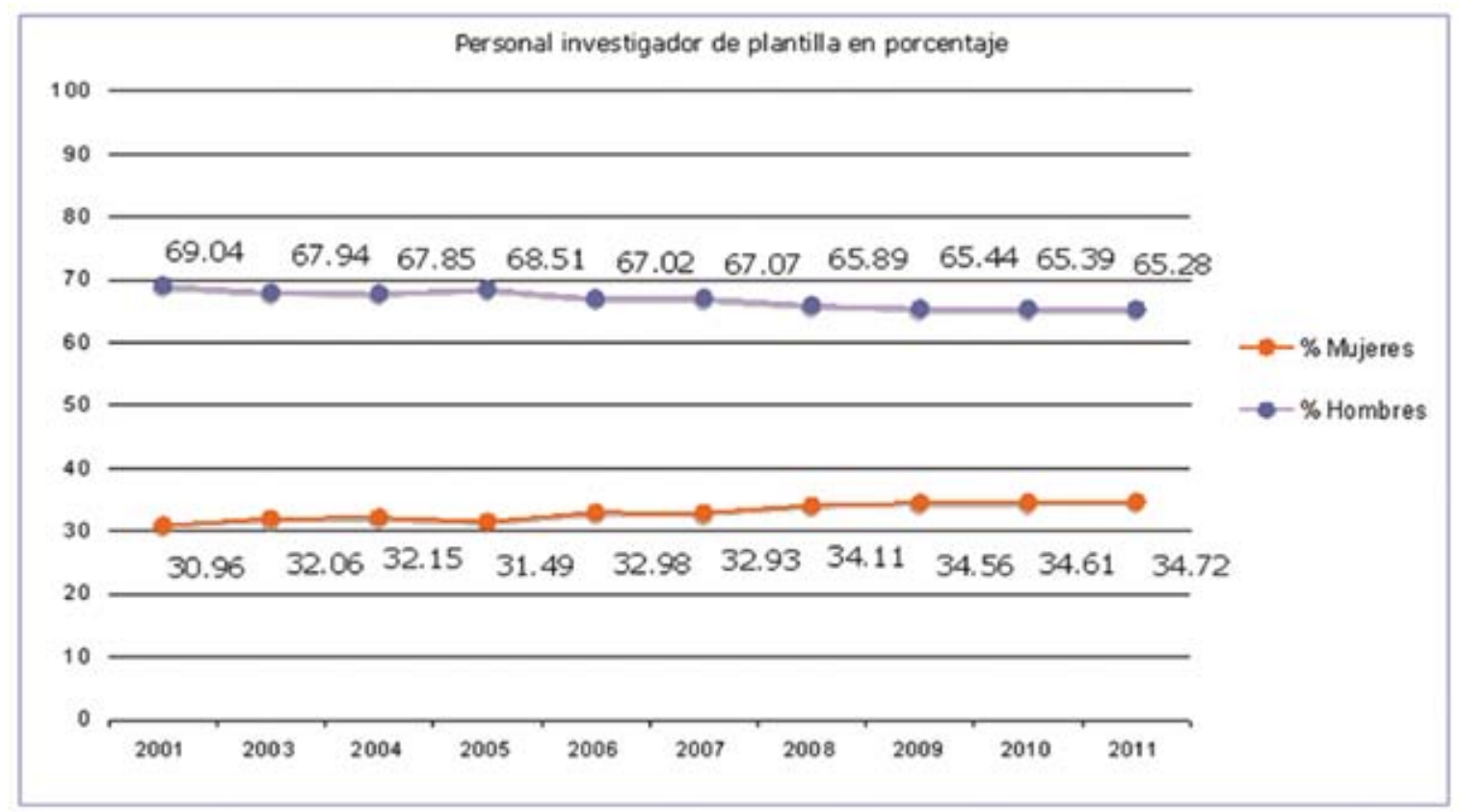


políticas a las instituciones y en su informe "Cambio Estructural de las Instituciones Científicas" se identifican como problemas comunes, la falta de transparencia en la toma de decisiones o la existencia de redes de amiguismo y de prejuicios que influyen en las valoraciones de los méritos del personal (UE, 2011). Estas consideraciones, unidas a la situación actual de recesión económica, en la que están sufriendo mayores retrocesos las mujeres, hacen que las políticas proactivas de igualdad sigan siendo imprescindibles.

\section{AGRADECIMIENTOS}

Las autoras de este artículo, miembros actuales de la Comisión Mujeres y Ciencia del CSIC, agradecen a todos los componentes pasados y presentes su trabajo en la Comisión, especialmente a Ma Luz Martínez Cano su gran trabajo como Secretaria de la misma desde 2008. Agradecemos también a Ana Robles (CIBCSIC) su inestimable ayuda en la elaboración de varias figuras.

\section{BIBLIOGRAFÍA}

Alcalá Cortijo, P. (1996) “Españolas en el CSIC. Presencia y status de las mujeres en la investigación científica española, 1940-1993". En T. Ortiz y G. Becerra (eds.), Mujeres de ciencias. Mujer, feminismo y ciencias experimentales y tecnológicas, Granada, Universidad de Granada, Feminae.

CSIC (2001). Secretaría General de Recursos Humanos, 2001. Informe Mujeres Investigadoras 2001, 15 pp. (http:// www.csic.es/web/guest/informes-precedentes1).

CSIC (2003). Subdirección General de Recursos Humanos, 2003. Informe: Mujeres investigadoras del CSIC 2003, 16 pp. (http://www.csic.es/web/guest/ informes-precedentes1).

CSIC (2012): Mujeres y Ciencia. Informe Mujeres Investigadoras CSIC 2012, 27 pp. (http://www.csic.es/web/guest/ informes-cmyc).

De Pablo, F. (2000). Mujer y ciencia desde la Europa del sur. El País (Futuro). 05/01/2000.

Duarte, C. (2009). "Igualdad de Género: ¿Y en ciencia cuándo?". El País, 28 de diciembre de 2009 (http://sociedad.elpais.com/sociedad/2009/12/28/actualidad/1261954814_850215.html).

EUROPEAN COMMISSION (2003). Third European Report on Science \& Technology Indicators. Luxembourg: Office for Official Publications of the European Communities.

Fernández Vargas, V. y Santesmases, M. J. (eds.) (2002). Ciencia y tecnología en el CSIC: una visión de género, Arbor vol. 172, número 679-680. Disponible en (http://arbor.revistas.csic.es/index. php/arbor/issue/view/84).
Flecha García, C. (1996). Las primeras universitarias en España 1872-1910, Editorial Narcea.

López Sancho, M. P. (2012a). "La excelencia científica y la perspectiva de género." RSEF septiembre 2012, (se puede descargar en: http://www. rsef.org/index. php?option=com content \&view $=$ article $\& i d=314: l a-$ excelencia-cientifica-y-la-perspectiva-de-genero\&catid=71:fisica-aldia\&ltemid=137).

López Sancho, M. P. (2012b): “Mujeres en Ciencia y Tecnología". Con la A, noviembre 2012. (http://revista.conlaa.com/).

Magallón Portolés, C. (1998/2005). Pioneras españolas en las ciencias. Las mujeres del Instituto Nacional de Física y Química, Estudios sobre la Ciencia no 24. Madrid: Consejo Superior de Investigaciones Científicas.

Moss-Racusin, C. A.; Dovidio, J. F.; Brescoll, V. L.; Graham, M. J. y Handelsman, J. (2012). "Science faculty's subtle gender biases favor males students", (www.pnas.org/cgi/doi/10.1073/ pnas.1211286).

Pereira, L. (2012). "Igualdad de Género", Carta al Director, El País, 2 de noviembre de 2012 (http://elpais.com/elpais/2012/11/01/opinion/1351785390_305001.html).

Pérez Sedeño, E. (coord.) (2003). La situación de las mujeres en el sistema educativo de ciencia y tecnología en España y en su contexto internacional", Programa de Análisis y estudios de acciones destinadas a la mejora de la Calidad de la Enseñanza Superior y de Actividades del Profesorado Universitario (REF: S2/EA2003-0031). Disponible en (http://www.csic.es/web/guest/ informes-precedentes1. Último acceso, 7 de enero de 2013).
Tarrach, R. (2002). “Presentación". En V. Fernández Vargas y M. J. Santesmases (eds.), Ciencia y tecnología en el CSIC: una visión de género, $A R B O R$, vol. 172, número 679-680, Tomo CLXXII.

UNIÓN EUROPEA (2001). Política Científica de la Unión Europea, Promover la excelencia mediante la integración de la igualdad entre géneros, Comisión Europea, Dirección General de Investigación, Luxemburgo, ftp://ftp.cordis. europa.eu/pub/improving/docs/g_wo_ etan_es 200101.pdf (último acceso: 24/06/2010).

UNIÓN EUROPEA (2003). She Figures, (disponible en http://ec.europa.eu/ research/science-society/pdf/she_figures_2003.pdf).

UNIÓN EUROPEA (2006). She Figures (disponible en http://kif.nbi.dk/She_Figures_2006.pdf).

UNIÓN EUROPEA (2009). She Figures, (disponible en http://ec.europa.eu/research/science-society/document_library/pdf_06/she figures_2009_en.pdf).

UNIÓN EUROPEA (2009). Stocking 10 years of women in science policy by the European Commission 19992009, (disponible en http://ec.europa. $\mathrm{eu} / \mathrm{research} / \mathrm{science}$-society/index. cfm?fuseaction=public.topic\&id=1622).

UNIÓN EUROPEA (2011). EU 24905 "Structural change in research institutions: Enhancing excellence, gender equality and efficiency in research and innovation", (disponible en http://ec.europa. eu/research/science-society/index. cfm?fuseaction=public.topic\&id=122).

Wenneras, C. and Wold, A. (1997). "Nepotism and Sexism in peer-review", Nature 387, 341. 


\section{ANEXO: Relación de MIEMBros de La Comisión MUJeres y CienCIA del CSIC DESIGNADOS POR LA PRESIDENCIA Y ELECTOS}

\section{Grupo de Trabajo (2001-2002, por designación de Presidencia)}

Preside Dr. Rolf Tarrach (Presidente del CSIC)

Vocales: Dra. Carmen Nieves Afonso, Dra. María Ángeles Durán, Dra. Valentina Fernández Vargas, Dra. Montserrat Gomendio, Dra. Flora de Pablo, Dra. Caridad Ruiz Valero, Dra. Matilde Sánchez Ayuso, Dra. Montserrat Torné, Dña. Yolanda Varela.

CMYC 2003 (desde entonces asisten vocales electas por las áreas científicas)

Preside Dra. Manuela Juárez (por delegación del Presidente Emilio Lora Tamayo)

Secretario: Dr. Alfredo Alvar

Vocales: Dra. Joaquina Álvarez Marrón, Dra. Alicia de Andrés, Dra. Flora de Pablo, Dra. Mar García Hernández, Dra. María Jesús Lázaro Elorri, Dra. Eulalia Pérez Sedeño, Dra. Carmen Polo, Dra. Francisca Sevilla, Dra. Montserrat Torné, Dña. Yolanda Varela, Dra. Carmen Vidal.

\section{CMYC 2004}

Preside Dra. Montserrat Torné (por delegación del Presidente Carlos Martínez)

Secretaria: Dra. Alicia de Andrés

Vocales: Dr. Alfredo Alvar, Dra. Joaquina Álvarez Marrón, Dra. Flora de Pablo, Dra. Mar García Hernández, Dra. María Jesús Lázaro Elorri, Dra. Eulalia Pérez Sedeño, Dra. Carmen Polo, Dra. Francisca Sevilla, Dra. Carmen Vidal.

\section{CMYC 2005}

Preside Dña. Paz Juárez o Dña. Rosario Martín Herranz (por delegación del Presidente Carlos Martínez)

Secretaria: Dra. Alicia de Andrés

Vocales: Dra. Joaquina Álvarez Marrón, Dra. Pilar Cano, Dra. Alicia de Andrés, Dra. Flora de Pablo, Dra. Mar García Hernández, Dra. María Jesús Lázaro Elorri, D. José Ramón Márquez, Dra. Eulalia Pérez Sedeño, Dra. Francisca Sevilla, Dra. Carmen Vidal.

\section{CMYC 2006}

Preside Dña. Rosario Martín Herranz (por delegación del Presidente Carlos Martínez)

\section{Secretaria: Dra. Joaquina Álvarez Marrón}

Vocales: Dra. Alicia de Andrés, Dra. Flora de Pablo, Dra. Mar García Hernández, Dra. María Jesús Lázaro Elorri, Dra. Victoria Moreno,
Dra. Mạ Teresa Ortega, Dra. Eulalia Pérez Sedeño, Dña. Sara Quiles, Dra. Francisca Sevilla, Dra. Carmen Vidal.

\section{CMYC 2007}

Preside Dña. Rosario Martín Herranz (por delegación del Presidente Carlos Martínez)

Secretaria: Dra. Joaquina Álvarez Marrón_

Vocales: Dra. Alicia de Andrés, Dra. Flora de Pablo, Dra. Mar García Hernández, Dra. María Jesús Lázaro Elorri, Dra. Pilar López Sancho, Dra. Victoria Moreno, Dra. Pilar Nieva, Dra. Ma Teresa Ortega, Dña. Sara Quiles, Dra. Francisca Sevilla.

\section{CMYC 2008}

Preside Dr. Rafael Rodrigo o Dra. Pilar López Sancho (por delegación del Presidente Carlos Martínez)

Secretaria: Dra. Joaquina Álvarez Marrón o Dña. Mạ Luz Martínez Cano

Vocales: Dra. Francesca Campabadal, Dra. Alicia Durán, Dra. Mar García, Dra. María Jesús Lázaro Elorri, Dra. Pilar López Sancho, Dra. Victoria Moreno, Dra. Pilar Nieva, Dra. Mạ Teresa Ortega, Dra. Francisca Sevilla.

\section{CMYC 2009-2011}

Preside Dra. Pilar López Sancho (por delegación del Presidente Rafael Rodrigo)

\section{Secretaria: Dña. Ma Luz Martínez Cano}

Vocales: Dra. Joaquina Álvarez Marrón, Dra. Francesca Campabadal, Dr. Felipe Criado, Dr. Juan José Damborenea, Dra. Flora de Pablo, Dra. Alicia Durán, Dra. María Jesús Lázaro Elorri, Dra. Elena Molina, Dra. Pilar Nieva, Dra. Luisa María Sandalio, Dra. Eulalia Pérez Sedeño.

\section{CMYC 2012-2013}

Preside Dra. Pilar López Sancho (por delegación del Presidente Emilio Lora Tamayo)

\section{Secretaria: Dña. Ma Luz Martínez Cano_}

Vocales: Dra. Joaquina Álvarez Marrón, Dra. Flora de Pablo, D. Jesús González Ayuso, Dra. Josefa Masegosa, Dra. Ma Carmen Mayoral, Dra. Elena Molina, Dra. Eulalia Moreno Mañas, Dra. Eulalia Pérez Sedeño, Dra. Francisca Puertas Maroto, Dr. Miguel Ángel Puig-Samper, Dra. Luisa Ma Sandalio. 